\title{
Using a new hybrid rootstock significantly increases the grafted plant rate and watermelon yield**
}

\author{
Jian Zhang ${ }^{1,2}$, Pengcheng Wang ${ }^{1,2}$, Hongmei Tian ${ }^{1,2}$, Yan Wang ${ }^{1,2}$, and Haikun Jiang ${ }^{1,2,3}$ \\ 'Institute of Horticulture, Anhui Academy of Agricultural Sciences, Hefei 230031 Anhui Province, China \\ ${ }^{2}$ Key Laboratory of Genetic Improvement and Ecophysiology of Horticultural Crops, Hefei 230031 Anhui Province, China \\ ${ }^{3}$ School of Horticulture, Anhui Agricultural University, Hefei 230036 Anhui Province, China
}

Received February 27, 2018; accepted August 7, 2018

\begin{abstract}
Rootstocks constitute a very important resource of grafted plants, especially in terms of improving the production of watermelon. In this work, a new rootstock, i.e. 'Wanzhen No. 2 ', was used to evaluate the grafting effect on three watermelon cultivars ('Xiuli', 'Jinlvhongling', and 'Xinong No. 8') subjected to double-root-cutting grafting. Protected field trials were also conducted. The obtained results have shown that the doubleroot-cutting grafting technique significantly increases the root length and dry weight of 'Wanzhen No. 2', when compared to bottle gourd rootstock. 'Wanzhen No. 2' was also found to significantly increase the average survival rate of grafted seedlings, up to $96 \%$ in three experimental bases. The fruits harvested from the 'Wanzhen No. 2' grafted plants had higher weight than those obtained from non-grafted plants, without affecting the central sugar content. By using 'Wanzhen No. 2' as the rootstock, the watermelon yield significantly improved in 'Xiuli' (by 26.7 and $87.4 \%$ ) and 'Wanzhen No. 2' (by 16.3 and 89.2\%), when compared to both the control 'Jiashi' and the non-grafted plant. The yield of 'Xinong No. 8' significantly increased (by 32.2\%), when compared to the non-grafted plant. Our study suggests that 'Wanzhen No. 2' is a suitable rootstock for grafted seedlings in three watermelon cultivars. We have provided new insights into the rootstock and contributed to the improvement of watermelon grafted plants.

Keywords: pumpkin, double root cutting, grafted plant, watermelon yield
\end{abstract}

\section{INTRODUCTION}

Watermelon is one of the world's most important vegetables. China has become the largest consumer of this crop (Ren et al., 2012). China is reportedly the leading global

*Corresponding author e-mail: microbiol@126.com pengchengah@hotmail.com

**This work was financially supported by the Fund of National Key Research and Development Program of China (2018YFD0201309) (2018 to 2020), the Anhui Science and Technology Project (1804h07020152 and 1704a07020078) (2018 to 2020) and the Vegetable Cultivation Scientific Innovation Team (18C0309) (2018 to 2020) from the Anhui Academy of Agricultural Sciences. watermelon producer, with approximately 1.8 million ha under cultivation in 2013 and with more than 1 million ha of watermelon annually grown in that country (Mo et al., 2016). However, one of the major problems encountered in watermelon production is the decrease in both fruit yield and quality caused by soil diseases (Huitrón-Ramírezet al., 2009), especially in the current plantations with a huge decrease in soil quality. Consequently, it has become of utmost importance to search for effective strategies that could lead to an improved plant tolerance to soil-borne diseases.

There are different ways to prevent the attacks of soil pathogens on plants, including crop rotation, genetic improvement and soil fumigation. However, each of these practices has certain inconveniences. For instance, the idea of growing new cultivars that would display strong resistance to soil-borne diseases is not only time consuming and expensive (Huitrón-Ramírez et al., 2009), but it has also proven futile because some pathogens are capable of quickly overcoming resistance (Lowe et al., 2011; Zhu et al., 2015). At present, grafting with resistant rootstocks offers one of the best ways to avoid soil diseases and improve plant growth. Grafting has recently become a basic support for the sustainable development of the modern vegetable industry in the Anhui Province (Wang et al., 2015).

The cultivation of grafted vegetables has been reportedly used since 1920 with the primary purpose of counteracting or diminishing attacks by soil pathogens, such as Fusarium, Verticillium and nematodes (Huang et al., 2009; King et al., 2008; Miguel et al., 2004). The grafting cultivation of vegetables can improve resistance to soil-borne

(C) 2019 Institute of Agrophysics, Polish Academy of Sciences 
diseases and enhance the overall resistance capacity. It also serves as a key technique to overcome the continuous cropping obstacle, and to keep the vegetable products safe and stable (King et al., 2008; Louws et al., 2010). Numerous researchers have emphasized that an interaction exists between rootstocks and scions, leading to an increased yield and fruit enhancement, due to large water and mineral uptake. For instance, Huitrón-Ramírezet al. (2009) reported that the use of watermelon grafted onto the 'RS841' and 'Shintosa Camelforce' rootstocks significantly increased the average fruit weight and the total watermelon yield. On the contrary, Romano and Paratore (2001) stated that vegetable grafting did not improve yield when rootstock selection was not suitable. Although grafting can negatively or positively affect the plant growth and yield, identifying a new rootstock for the production of vegetables appears important (Edelstein et al., 2014). However, to the best of our knowledge, new information about rootstocks remains scarce.

Therefore, the objective of this study was to evaluate the influence of a new hybrid pumpkin, namely 'WanZhen No. 2' ('WZ2'), subjected to the double-root-cutting grafting method, on watermelon in terms of its agronomic performance, yield and fruit quality attributes.

\section{MATERIAL AND METHODS}

This work was conducted at the experimental bases of the Institute of Horticulture at the Anhui Academy of Agricultural Sciences. The grafted experiments under greenhouse conditions were conducted in three cities (Fig. 1), namely Suzhou $\left(\mathrm{E} 116^{\circ} 09^{\prime}, \mathrm{N} 33^{\circ} 18^{\prime}\right)$, Huainan (E116 $21^{\prime}$, $\left.\mathrm{N} 32^{\circ} 23^{\prime}\right)$ and Huangshan $\left(\mathrm{E} 118^{\circ} 01^{\prime}, \mathrm{N} 30^{\circ} 01^{\prime}\right)$, in order

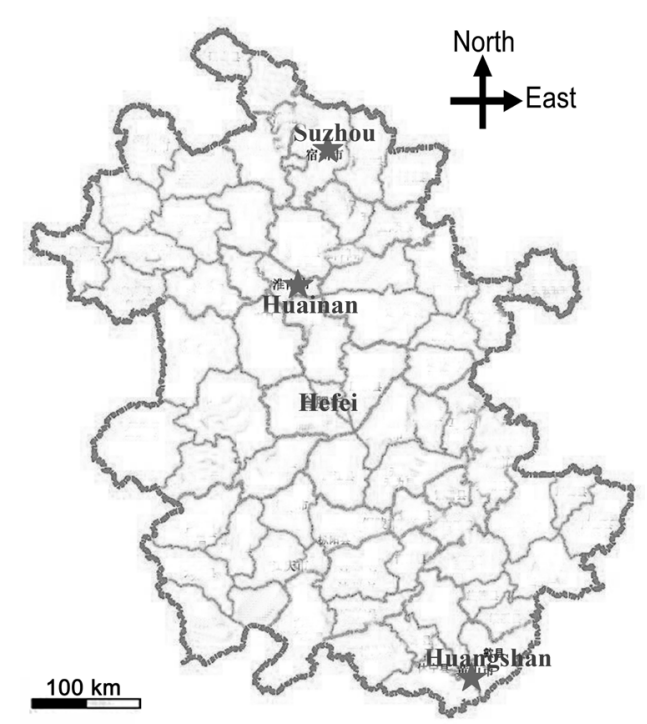

Fig. 1. Location of the three places selected for this study. The grafted experiments under greenhouse conditions were conducted in three cities, namely Suzhou (E116 $09^{\prime}$, N33 $\left.18^{\prime}\right)$, Huainan $\left(\mathrm{E} 116^{\circ} 21^{\prime}, \mathrm{N} 32^{\circ} 23^{\prime}\right)$ and Huangshan $\left(\mathrm{E} 118^{\circ} 01^{\prime}, \mathrm{N} 30^{\circ} 01^{\prime}\right)$. to evaluate the horticultural performance of watermelons grafted onto 'WZ2' serving as a new rootstock in the period from 1 January 2015 to 1 August 2016.

Three hybrids of watermelon (Citrullus lanatus), namely mini-fruit watermelon 'Xiuli' (from the Anhui Agriculture Horticulture Institute; single fruit weight, 2-3 kg; abbreviated as ' $\mathrm{W} 1$ '), medium-fruit watermelon 'Jinlvhongling' (from the Anhui Agriculture Horticulture Institute; single fruit weight, 5-8 kg; 'W2'), and largefruit watermelon 'Xinong No. 8' (obtained from Fengle Seed Co., Ltd., Hefei, China; single fruit weight, 8-9 kg; 'W3'), were used as the scion and were simply classified in accordance with the fruit weight. These cultivars are widely grown in the commercial production area of watermelon in the Anhui Province, China. A new pumpkin (Cucurbita moschata) rootstock, namely 'WZ2,' identified as a new hybrid variety in our research group, was used in this study. This cultivar is one of the most popular rootstocks available for watermelon grafting in the Anhui Province. The 'Jiashi' pumpkin rootstock and the bottle gourd (Lagenaria siceraria) rootstock, commonly referred to as the white gourd, were used as controls.

Three hybrids of watermelon and rootstock seeds were sown in rectangular flat plates and trays containing 50 holes, respectively. The substrate was made up of peat, vermiculite and perlite, with a volume ratio of $3: 1: 1$. The mixed substrates were sieved using a $5 \mathrm{~mm}$ sieve after being dried and smashed. The bulk density of the resultant substrate was $0.23 \mathrm{~g} \mathrm{~cm}^{-3}$, the total porosity was $85.8 \%$ (Ling et al., 2012), and the $\mathrm{pH}$ value was adjusted between 6.2 and 6.8. In accordance with the volume ratio of $1: 10$, the electrical conductivity was $0.19 \mathrm{mS} \mathrm{cm}$. The watermelon seeds were soaked in water for $12 \mathrm{~h}$ and subsequently sown in a seedling plate with a density of 3000 seeds $\mathrm{m}^{-2}$ under greenhouse conditions. The seeds of the rootstocks were germinated and sown in 50-cell Styrofoam trays, with the former substrate under greenhouse conditions, 1 week before watermelon seed sowing. The environmental conditions for germination were $24-28^{\circ} \mathrm{C}$ (temperature) and $50-70 \%$ (relative humidity) (Mohamed et al., 2014).

Consistently with former descriptions, three types of watermelon scions were prepared from ' $\mathrm{W} 1$,' 'W2' and 'W3.' The 'WZ2' (the white gourd) and 'Jiashi' rootstocks were used in the stage of two unfolded true leaves, and scions were left to attain that stage as well. The double-root-cutting method, which comprised the slide tongue method (Mohamed et al., 2014), was used on all rootstocks, which means that the scion and rootstock roots were both cut off (Fig. S1a). Scions and rootstocks were fixed by means of a plastic clip. After grafting, the plants were cultivated at a temperature of $25-28^{\circ} \mathrm{C}$, and humidity was kept at more than $95 \%$ for 3 days of healing (Fig. S1d). Then, the relative humidity was gradually lowered (from 95 to $40 \%$ ), whereas 
light intensity increased (from 0 to $80000 \mathrm{~lx}$ ) (Turhan et al., 2012). Furthermore, 500 seeds were used for each individual treatment, which was repeated three times.

With a view to evaluating the efficiency of the doubleroot-cutting methods, the 'WZ2' pumpkin rootstock was selected, the white gourd was used as a control rootstock, and 'W2' was set as the scion. Four treatments were arranged on the basis of the 'WZ2' rootstock to evaluate the optimal inserting depth in the grafted seedlings, which were 1-2, $2-3,3-4$, and $4-5 \mathrm{~cm}$. Grafted plants and treatments were transferred to the greenhouse under a normal management procedure. The environmental conditions for cultivation were $18-20^{\circ} \mathrm{C}$ at night and $25-28^{\circ} \mathrm{C}$ at daytime, and humidity ranged between 50 and $90 \%$. The cultivation process involved surface irrigation and normal fertilization.

The grafting rate of seedlings was calculated for each of the three cities (Fig. 1). Successively grafted plants were counted within 15 days after grafting. Fifty grafted plants per each greenhouse unit with triplets were used to individually measure the survival rate of the grafted seedlings. The plant heights and root lengths of the grafted seedlings were measured within 20 days. Root dry weights were determined by drying each separate plant material in an oven at a temperature of $70^{\circ} \mathrm{C}$ until the weight remained constant, and then the root dry weights were recorded with triplets.

A protected field experiment was conducted in Huainan, due to its location in the central region of the Anhui Province, in the planting area of watermelon. The local climate is temperate; summers are hot and rainy with total average rainfalls of 865.5 and $663 \mathrm{~mm}$ in 2015 and 2016, respectively. The soil is of the yellow fluvo-aquic type, and its basic physicochemical properties are as follows: the organic matter $2.0 \%$; the available nitrogen $284.21 \mathrm{mg} \mathrm{kg}^{-1}$; the available phosphorus $68.99 \mathrm{mg} \mathrm{kg}^{-1}$; and the available potassium $285.50 \mathrm{mg} \mathrm{kg}^{-1}$. Both the grafted and non-grafted seedlings were transplanted at the beginning of March under protected field conditions in a period of 5 years of continuous soil cropping. The normal and regular management was employed to foster seedling growth. The seedling death rate and plant growth performance were recorded for ten plants and five fruits. The experiment was completed at end of July. Watermelons of the 'W1' and 'W2' types matured within 72 days from planting, and those of the 'W3' type after 80 days. After watermelon maturation, a random selection of 50 seedlings was performed individually, in order to calculate the disease occurrence with triplets, by means of the following formula:

$$
\text { Disease occurrence }(\%)=\frac{\text { Number of diseased plants }}{\text { Number of total plants }} \times 100 \% \text {. }
$$

Several harvests were performed between 23 May and 30 June 2015, and 20 June 2016. The average yield from $667 \mathrm{~m}^{2}$ was recorded immediately after harvest. Five fruits from each replicate were randomly selected to assess fruit weight $(\mathrm{kg})$ and the soluble solid content of fruit juice (\%). Fruit attributes and properties were detected in accordance with the previous methods to assess the quality of the watermelon fruit, i.e. the fruit set rate, the abnormal fruit rate, rind thickness, the central sugar content, the yellowish bands in the flesh and fruit weight (Kolayli et al., 2010). In brief, the selected fruits were sliced, and the rinds and seeds were removed. Juice was extracted from each fruit, and the soluble solid concentration was determined using a hand refractometer at $20^{\circ} \mathrm{C}$ (Precision and Scientific Instrument Co., Ltd., Changzhou, China). Fifty plants were used for both non-grafted and grafted plants with three replicates.

A randomized complete block was used as the field experimental design. Each treatment (plant material) had three blocks, and data were presented as mean values $(n \geq 5)$. Field data concerned an average period of 2 years. The analysis was conducted using the one-way (ANOVA) procedure. Means were separated through Fisher's protected LSD test, at $\mathrm{p}<0.05$, followed by Duncan's Test, at $p<0.05$. Statistical analyses were conducted using SPSS software (version 19.0, Inc., Chicago, Illinois, EUA).

\section{RESULTS}

The results showed that the survival rates of the grafted seedlings for 'W1' were 94.8, 95.7 and 97.6\% in Suzhou, Huainan and Huangshan, respectively, which increased by

Table 1. Grafted survival rate of watermelon plants under greenhouse condition

\begin{tabular}{cccccc}
\hline \multirow{2}{*}{ Watermelon } & Treatment & \multicolumn{4}{c}{ Grafted survival rate (\%) } \\
\cline { 3 - 6 } & & Suzhou & HuaiNan & HuangShan & Average \\
\hline \multirow{2}{*}{ W1 } & 'Jiashi' & $86.81 \pm 0.71$ & $90.71 \pm 0.54$ & $91.43 \pm 0.55$ & $89.69 \pm 0.63$ \\
& 'WZ2' & $94.86 \pm 0.85$ & $95.75 \pm 0.86$ & $97.62 \pm 0.98$ & $96.05 \pm 0.86$ \\
W2 & 'Jiashi' & $87.52 \pm 0.70$ & $91.31 \pm 0.91$ & $89.70 \pm 0.72$ & $89.52 \pm 0.63$ \\
& 'WZ2' & $98.61 \pm 0.79 *$ & $96.90 \pm 0.78$ & $97.11 \pm 0.78$ & $97.56 \pm 0.98$ \\
W3 & 'Jiashi' & $87.20 \pm 0.52$ & $89.82 \pm 0.45$ & $92.17 \pm 0.55$ & $89.71 \pm 0.63$ \\
& 'WZ2' & $95.33 \pm 0.86$ & $96.14 \pm 0.86$ & $98.20 \pm 0.88$ & $96.54 \pm 0.77$ \\
\hline
\end{tabular}

Grafted survival rate of mini-fruit watermelon 'Xiuli', W1, medium-fruit watermelon 'Jinlvhongling', W2, large fruit watermelon 'Xinong No. 8', W3, 'Jiashi', control treatment; analysis was achieved by using a one-way analysis of variance procedure (ANOVA), means $\pm \mathrm{SD}$, *significant at $\mathrm{p}<0.05$. 
$9.2,5.5$ and $6.8 \%$, respectively, when compared to the controls (Table 1). The survival rates of the grafted seedlings for 'W2' were 98.6, 96.9 and 97.1\% in Suzhou, Huainan and Huangshan, respectively, which increased by $12.7 \%$ (*significant at $\mathrm{p}<0.05$ level), 6.1 and $8.2 \%$, respectively, when compared to the controls. The survival rates of the grafted seedlings for ' $W 3$ ' were 95.3, 96.1 and 98.2\% in Suzhou, Huainan and Huangshan, respectively, which increased by $9.3,7.0$, and $6.6 \%$, respectively, when compared to the controls. The average survival rates for ' $\mathrm{W} 1$ ', 'W2' and 'W3' were 96, 97.5 and 96.5, respectively.

Under the same greenhouse conditions, watermelon grafted onto the 'WZ2' rootstock promoted the seedlings plant height, whereas the 'Jiashi' rootstock roots proved to be weak (Fig. 2A). The plant height and root length in 'Jiashi' were 10.1 and $13.7 \mathrm{~cm}$, respectively. The'WZ2' grafted seedlings height and root length were 15.1 and $16.8 \mathrm{~cm}$, respectively, which significantly raised the plant height and root length by $49.1 \%$ and $22.0 \%$ ( $p \leq 0.05$ ), respectively, when compared to the white gourd rootstock grafted plants. A developed root system was observed in the 'WZ2' treatment (Fig. 3A). The dry root weight from 'WZ2' was $0.21 \mathrm{~g}$, whereas the white gourd root dry weight was only $0.16 \mathrm{~g}$ (Fig. 3B). The use of 'WZ2' significantly increased the root dry matter by $31.2 \%$.

The inserting depth affected root growth. Under the same management conditions, not only the longest total root length was observed in the $2-3 \mathrm{~cm}$ depth but also the most developed root system was detected for this treatment (Fig. 4A). The total root lengths increased to 16.8, 17.6,
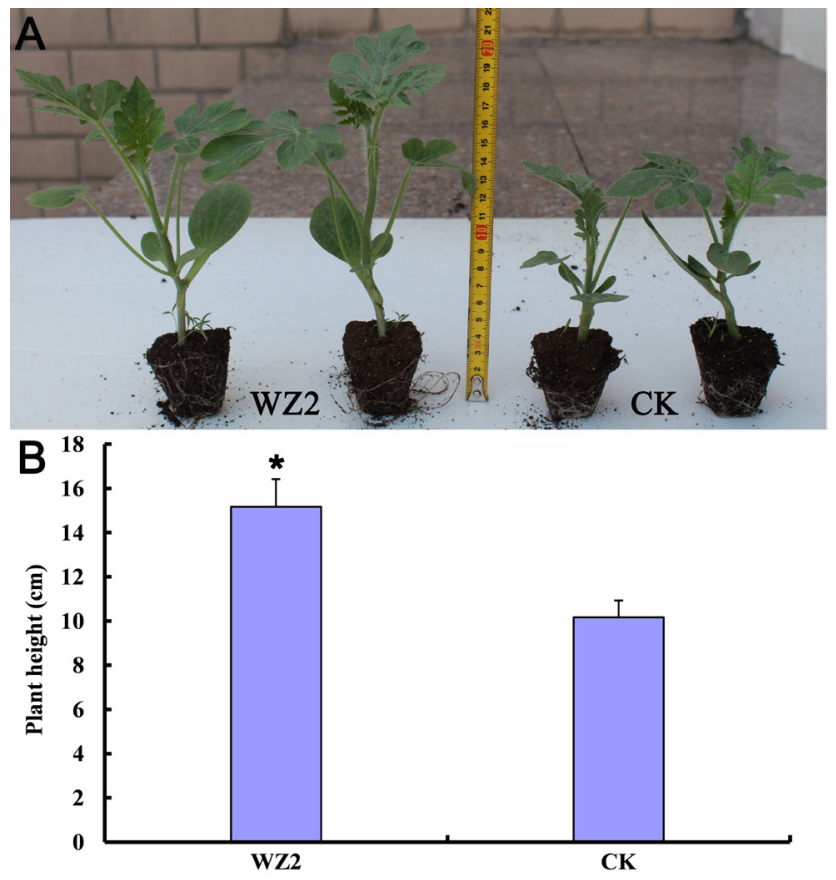

Fig. 2. Photo of plant growth performance in the 'WZ2' grafted plant and control treatment. The analysis was performed using a one-way ANOVA procedure, at $\mathrm{p}<0.05$.

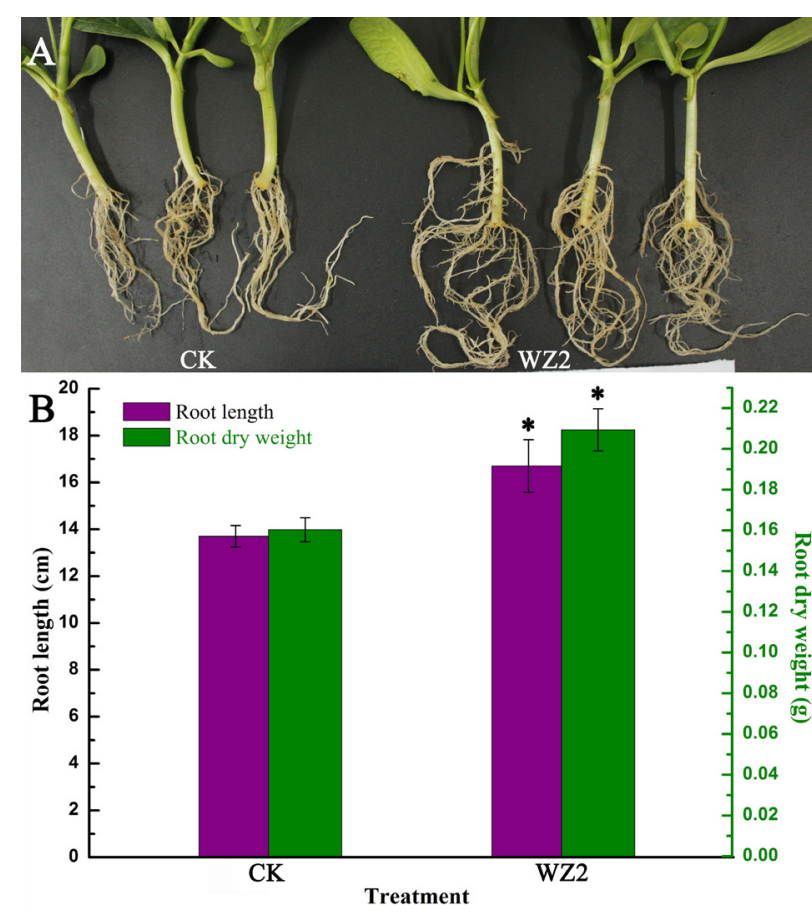

Fig. 3. Photo of root growth, length and dry weight in the 'WZ2' grafted plant and control treatment. The analysis was performed using a one-way ANOVA procedure, at $\mathrm{p}<0.05$.
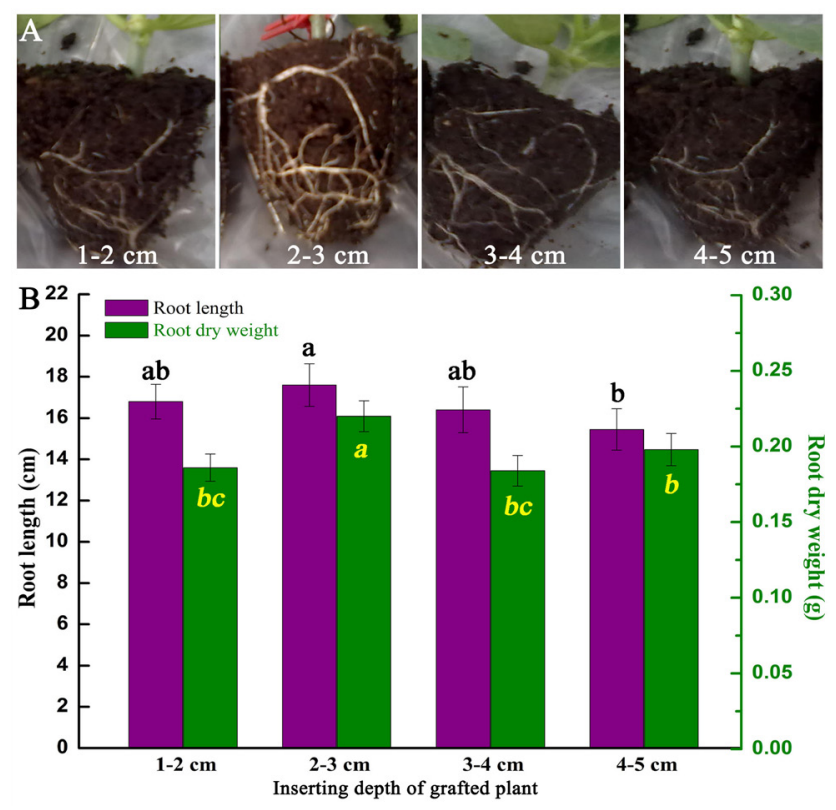

Fig. 4. Evaluation of root length and dry weight for different inserting depths of the 'WZ2' grafted plant. The analysis was performed by means of Duncan's test, at $p<0.05$, where $a, b$ and $c$ mean that two groups displayed significant differences, and $a b$ and bc indicate differences that were not significant. 
16.4 and $15.4 \mathrm{~cm}$ at depths of 1-2, 2-3, 3-4 and 4-5 cm, respectively (Fig. 4B). The total root length in $2-3 \mathrm{~cm}$ significantly increased by $13.9 \%(\mathrm{p}<0.05)$, when compared to that in $4-5 \mathrm{~cm}$ treatments, and by 4.7 and $7.3 \%$, when compared to that in 1-2and 3-4 cm treatments, respectively. The dry weight of roots was the largest at 2-3 cm, i.e. $0.22 \mathrm{~g}$, whereas at 1-2, 3-4 and 4-5 cm it amounted to $0.186,0.184$ and $0.198 \mathrm{~g}$, respectively (Fig. 4B). The most suitable inserting depth for ' $W Z 2$ ' was $2-3 \mathrm{~cm}$, which allowed for root regeneration.

The results obtained from the protected field experiment showed that no plant death was detected in the ' $\mathrm{W} 1$ ', 'W2' and 'W3' grafted plants when the 'WZ2' pumpkin was used as a rootstock. However, for ' $\mathrm{W} 1$ ', the non-grafted seedlings death rate reached $36.8 \%$, whereas for the 'Jiashi' control rootstock, the grafted seedlings death rate was $14.2 \%$. For ' $W 2$ ', the non-grafted seedlings death rate increased up to $30.3 \%$, whereas for the 'Jiashi' control rootstock, the grafted seedlings death rate was $7.3 \%$. For ' $\mathrm{W} 3$ ', the non-grafted seedlings death rate increased up to $29.8 \%$, whereas for the 'Jiashi' control rootstock pumpkin, the grafted seedlings death rate was $7.6 \%$ (Table 2).

For the three types of watermelon, the growth performance of the plants grafted onto the 'WZ2' rootstock was weaker than that of the control 'Jiashi', but it was stronger than that of the non-grafted seedlings (Table 2). For 'W1', the'WZ2' rootstock seedling fruit setting rate was close to the values obtained for the non-grafted seedlings, which amounted to 96.4 and $96.1 \%$, whereas the common pumpkin fruit setting rate was only $87.7 \%$, which was significantly lower than that of 'WZ2' (Table 3 ). The 'WZ2' rootstock abnormal fruit rate was only $1.8 \%$, which was significantly lower than the values obtained for both the 'Jiashi' control rootstock and non-grafted seedlings. For 'W2', the 'WZ2' rootstock seedling fruit setting rate was close to the values obtained for the non-grafted seedlings, which amounted to 96.7 and $96.1 \%$, whereas the common pumpkin fruit setting rate was only $87.4 \%$, which was significantly lower than that of 'WZ2'. The 'WZ2' rootstock abnormal fruit rate was only $3.3 \%$, which was significantly lower than the rate of $24.5 \%$ determined for the 'Jiashi' control rootstock, but it was higher than that of the non-grafted seedling $(2.5 \%$.) For ' $W 3$ ', the 'WZ2' rootstock seedling fruit setting rate was close to the values obtained for the non-grafted seedlings, which amounted to 96.4 and $95.4 \%$, whereas the common pumpkin fruit setting rate was only $87.7 \%$, which was significantly lower than that of 'WZ2'. The 'WZ2' rootstock abnormal fruit rate was $8.3 \%$, which was significantly lower than that of the 'Jiashi' control rootstock (17.9\%), but it was higher than that of the non-grafted seedlings $(7.7 \%)$.

Rind thickness was considerably higher in both grafted plants form the 'Jiashi' and 'WZ2' rootstocks, whereas the non-grafted seedlings rind thickness was the thinnest among the three cultivars (Table 3). The 'Jiashi' grafted plants showed a high rind thickness in both 'W1' and 'W2'. The 'WZ2' grafted plants showed nearly the same rind thickness as the non-grafted plants. The yellowish bands in the flesh were light in the 'WZ2' grafted plants, and the same was observed for the non-grafted plants in three watermelon varieties. However, the 'Jiashi' control rootstock grafted plants showed a high degree of yellow flesh.

For the 'W1' and 'WZ2' rootstocks, the central sugar content was consistent with the values obtained for the non-grafted seedlings, which amounted to 13.2 and 13.1, and it was significantly higher than that of the control 'Jiashi', which was 11.8. For the 'W2', the 'WZ2' rootstocks, the central sugar content was also consistent with the values obtained for the non-grafted seedlings, which amounted to 12.2 and 12.4, and it was significantly higher than that of the control 'Jiashi', which was 10.6. For 'W3', the 'WZ2' rootstock central sugar content was lower than

Table 2. Qualitative plant growth parameters and disease rate of nongrafted and grafted watermelon plants in protected field experiment

\begin{tabular}{|c|c|c|c|c|}
\hline \multirow{2}{*}{ Watermelon } & \multirow{2}{*}{ Treatment } & \multicolumn{3}{|c|}{ Plant growth parameters } \\
\hline & & PGP & SDR (\%) & $\operatorname{DIR}(\%)$ \\
\hline \multirow{3}{*}{ W1 } & nongrafted & weak & $36.8 \pm 1.2 \mathrm{a}$ & $37.1 \pm 2.1 \mathrm{a}$ \\
\hline & 'Jiashi' & strong & $14.2 \pm 1.1 \mathrm{~b}$ & $15.3 \pm 1.7 \mathrm{~b}$ \\
\hline & 'WZ2' & medium & $\mathrm{ND}^{*}$ & ND \\
\hline \multirow{3}{*}{ W2 } & nongrafted & weak & $30.3 \pm 2.1 \mathrm{a}$ & $25.8 \pm 3.1 \mathrm{a}$ \\
\hline & 'Jiashi' & strong & $7.3 \pm 1.6 b$ & $10.7 \pm 1.9$ \\
\hline & 'WZ2' & medium & ND & ND \\
\hline \multirow{3}{*}{ W3 } & nongrafted & weak & $29.8 \pm 1.8 \mathrm{a}$ & $26.8 \pm 2.2 \mathrm{a}$ \\
\hline & 'Jiashi' & strong & $7.3 \pm 1.4 \mathrm{~b}$ & $12.5 \pm 1.6 b$ \\
\hline & 'WZ2' & medium & ND & ND \\
\hline
\end{tabular}

PGP - plant growth performance, SDR - seedling death rate, DIR - disease rate, *ND - not detected; a, b, c - means two groups having significant; $a b, b c$ - means having difference but not significant. 


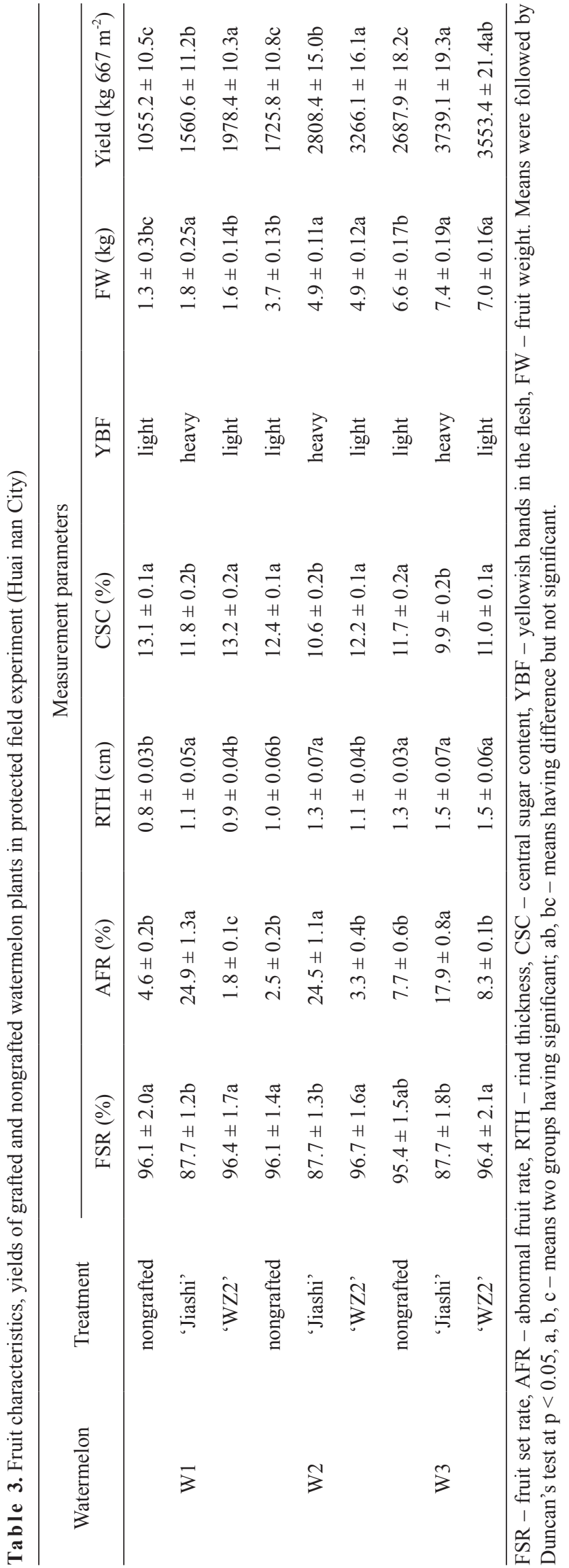

the values obtained for the non-grafted seedlings, which amounted to 11.0 and 11.7 , and it was significantly higher than that of the control 'Jiashi', which was 9.9 (Table 3).

For ' $\mathrm{W} 1$ ', the average weight of the 'WZ2' rootstock was $1.6 \mathrm{~kg}$, which was higher than that of the non-grafted plants $(1.3 \mathrm{~kg})$ but lower than that of the 'Jiashi' control rootstock $(1.8 \mathrm{~kg})$. Watermelon yield was $1978.4 \mathrm{~kg} 667 \mathrm{~m}^{-2}$, which increased by 26.7 and $87.4 \%$, respectively, when compared to the control 'Jiashi' and the non-grafted seedlings. For 'W2', the average weight of the 'WZ2' rootstock was $4.9 \mathrm{~kg}$, which was higher than that of the non-grafted seedlings $(3.7 \mathrm{~kg})$, and consistent with the 'Jiashi' control rootstock $(4.9 \mathrm{~kg})$. The use of the ' $\mathrm{WZ2}$ ' rootstock increased the watermelon yield to $3266.1 \mathrm{~kg} 667 \mathrm{~m}^{-2}$, which was by 16.3 and $89.2 \%$ higher when compared to both the control 'Jiashi' and the non-grafted seedlings. For 'W3', the average weight of the 'WZ2'rootstock was $7.0 \mathrm{~kg}$, which was higher than that of the non-grafted seedlings $(6.6 \mathrm{~kg})$, and slightly lower than that of the 'Jiashi' control treatment rootstock $(7.4 \mathrm{~kg})$. The use of the 'WZ2' rootstock increased the large-fruit-type watermelon yield by $3553.4 \mathrm{~kg}$ $667 \mathrm{~m}^{-2}$, which was significantly (i.e. by $32.2 \%$ ) higher, when compared to the non-grafted seedlings. However, the 'Jiashi' rootstock treatment yield was $3739.1 \mathrm{~kg} 667$ $\mathrm{m}^{-2}$, which increased by $5 \%$, when compared to 'WZ2' (Table 3). Therefore, although no significant difference was detected in fruit quality, the yield of 'WZ2' was different, when compared to both the control 'Jiashi' and the non-grafted plants.

During the protected field trials conducted between 1 January 2015 and 1 August 2016, no plant disease caused by Fusariumoxysporum was detected in the three types of watermelon, in which the plants were grafted onto the 'WZ2' rootstock, even though the plants grew in 5 years of continuous soil cropping (Table 2). However, high disease rates were detected in the non-grafted plants, i.e. 37.1, 25.8 , and $26.8 \%$ for ' $\mathrm{W} 1$ ', 'W2' and 'W3', respectively. However, the disease rates in the control-grafted plant 'Jiashi' were 15.3, 10.7 and $12.5 \%$ for 'W1', 'W2', and 'W3', respectively.

\section{DISCUSSION}

Grafting is an important technique to improve plant growth, biotic or abiotic stress resistance, as well as productivity, especially in the continuous soil cropping patterns in the cultivation of watermelon (King et al., 2010; Lee et al., 2010; Miguel et al., 2004). Therefore, new rootstocks are valuable resources to cope with the increasing soil diseases, especially in intensive planting areas. Our studies indicate that the pumpkin 'WZ2' rootstock can increase the yield of the three types of watermelon. At present, most of the cucurbit rootstocks are bottle gourd cultivars (King et al., 2010), and a few new types are being tested for grafts in 
the pumpkin rootstock. These results indicate that the new pumpkin rootstock 'WZ2' is a possible rootstock resource for watermelon grafting.

The survival rate of the grafted seedling is an important criterion for successfully grafted plants, not only for watermelons but also for other important vegetables $(\mathrm{Hu}$ et al., 2016; Lee et al., 2010; Vu et al., 2014). By using the 'WZ2' rootstock, the survival rate was found to significantly increase in the three types of watermelon, as compared to the normal 'Jiashi' rootstock in the three cities under analysis. The highest survival rates of the grafted seedlings of both 'W1' and 'W2' were found in Suzhou, whereas the highest survival rates of the grafted 'W3'seedling was found in Huangshan. $\mathrm{Vu}$ et al. (2014) reported that the survival rate was improved by controlling environmental factors, such as temperature and humidity, during the healing and acclimatization period. In our study, all the grafted seedlings, including those intended for treatments, were placed under the same cultivation conditions, and no significant difference was found among the three experiment bases when 'WZ2' was used, suggesting that 'WZ2' improved the performance and stability parameters in different locations. On the contrary, significant differences in the control treatments of the normal 'Jiashi' rootstock were identified.

Watermelon can be grafted onto the bottle gourd variety (Yang et al., 2016). Here, we proved that scions from watermelon could also be grafted onto the newly selected pumpkin rootstock 'WZ2' (Fig. 2A), suggesting that the pumpkin rootstock was also an excellent option for watermelon-grafted seedlings. The results of this study were in accordance with the results presented by Xing et al. (2015) who reported that the grafting of cucumber seedlings with the pumpkin rootstock was a potential way to exhibit a strong ability to adapt to inconvenient environmental conditions. Here, we found that watermelon grafted onto the 'WZ2' rootstock significantly increased the grafted seedlings plant height (Fig. 2A), while also showing a robust root system (Fig. 3A), and root length and dry weight (Fig. 3B). Similar results were also reported by Xing et al. (2015), proving that pumpkin had a vigorous root system and could be used as a rootstock to promote the growth of cucumber seedling. The possible reasons were that plants grafted onto pumpkin were associated with high root dry weights, which presumably facilitated the uptake of nutrients by the plants (Savvas et al., 2010). A recent study has also proven that pumpkin rootstock grafting can increase watermelon plant performance under abiotic stress conditions (Huang et al., 2016).

When considering the grafting method, a previous study reported that the tongue approach grafting method seems to be the best technique for watermelon (Mohamed et al., 2014). In this work, a double-root-cutting grafting technique, combined with tongue approach grafting for scion, was adopted. The root from the pumpkin rootstock was cut off for regeneration (Fig. S1b). However, neither details of the double-root-cutting method nor the best depth for root growth on 'WZ2' were presented. Hence, four inserting depths were arranged to optimize the most suitable condition for the double-root-cutting method. We found that the best depth for grafted seedlings was 2-3 cm. Under such treatment, the regenerated root showed not only the longest length but also the highest dry weight (Fig. 4), suggesting that the inserting depth of the grafted seedling affected root growth. We presumed that the depth of $2-3 \mathrm{~cm}$ generated the best humidity and temperature for germinated roots. This study also confirmed that the double-root-cutting grafting technique could also regenerate a well-developed root system (Figs 3 a and S1c), and had the advantage of relative white gourd rootstock, exhibiting that the double-root-cutting method is significantly suitable for the 'WZ2' pumpkin rootstock. Thus, this attempt might reflect a useful way to cultivate high-quality grafted seedlings.

The use of 'WZ2' exhibited a better performance in the protected field trials than the control treatments, namely the 'Jiashi' rootstock, regardless of watermelon types. No seedling death was detected among 'W1', 'W2' and 'W3' when the three types of watermelon were grafted onto the 'WZ2' rootstock, indicating that this rootstock had an improved ability to resist soil diseases. On the contrary, seedling death was found in both the control 'Jiashi' and the non-grafted seedlings, with the highest seedling death rate being found in the latter type (Table 2). Our field trial results were consistent with previous studies reporting that non-grafted watermelons were sensitive to soil diseases (King et al., 2008; Ren et al., 2015) and confirming that non-grafted seedlings were easily attacked by soil-borne pathogens.

Fruit thickness was significantly affected by the rootstock's being used under protected field conditions. Both rootstocks increased the rind thickness, when compared to the non-grafted plants (Table 3), suggesting that grafting could affect the watermelon fruit property. However, the effect of grafting on the soluble solids of fruits was in accordance with the watermelon cultivars. In our study, the non-grafted seedlings had the highest central sugar content in all three watermelon cultivars. However, no significant difference in central sugar content existed when the three watermelons were grafted onto 'WZ2', suggesting that grafting onto 'WZ2' did not affect sugar accumulation. Here, the fruits harvested from the control 'Jiashi' showed the lowest central sugar content (Table 3). Previous studies reported that grafting might have adverse effects on fruit quality, especially when different rootstocks were used (Reig et al., 2016; Trionfetti et al., 2002). On the contrary, in our study, no significant detrimental effects of grafting on fruit quality were detected in the 'WZ2' grafted plants, such as no increase of the yellowish bands in the flesh or a dramatic reduction in sugar content. We have, therefore, presumed that 'WZ2' may have a strong affinity to the tested watermelons. Similar results were also reported by Miguel et al. (2004) who claimed that the use of a given rootstock had 
no effect on the soluble solid concentration. Furthermore, Edelstein et al. (2014) also reported that, although rootstocks had an inconsistent effect, e.g., a decrease in sugar content or an increase relative to the non-grafted controls, minimal or no differences were revealed at harvest in the central sugar content between the non-grafted and grafted watermelons. Our results were consistent with the formerly drawn conclusions that central sugar content depended on the watermelon cultivars and rootstock that had been used. However, in some cases, soluble solid contents were changed due to either environmental conditions or harvest time (Dorey et al., 2016).

For the fruit yield, in our study, which was conducted in a period of 5 years of continuous soil cropping, the yield of the most grafted watermelons significantly increased in the three types of watermelon, when compared to the control 'Jiashi' treatments as well as with the non-grafted plants under field conditions. Similar results were also reported by Turhan et al. (2012) who stated that the use of inter-specific hybrid pumpkin rootstocks significantly influenced watermelon fruit weight and total yield by grafting. Huitrón-Ramírez et al. (2009) also reported that the use of watermelon grafted onto the Cucurbita rootstock significantly increased the average fruit weight and the total yield of watermelon. Moreover, Proietti et al. (2008) also proved that grafting onto the rootstock could increase yield in the grafted watermelons. Our study confirmed that using 'WZ2' as a rootstock to produce grafted plants significantly increased the watermelon yield, suggesting that the pumpkin rootstock had the same potential ability as the bottle gourd rootstock.

The primary reasons for watermelon grafting are connected not only with better Fusarium resistance but also with an increased tolerance to salinity, cold, root-knot nematodes and, in some cases, viruses (King et al., 2010; Yang et al., 2013). Previous studies have proven that rootstock can influence disease resistance as well as yield in watermelon (Yetışır et al., 2003). Grafted seedlings from 'WZ2' showed resistance to $F$. oxysporum under the continuous soil cropping pattern, suggesting that 'WZ2' displayed a potential disease resistance, when compared to the control 'Jiashi' treatment, even if the grafted seedlings were grown in the same soil. Thus, we drew a conclusion that watermelon grafted onto the pumpkin 'WZ2' rootstock increased the yield, which is generally attributed to the enhancement of the root system, resulting in the improvement of water and plant nutrient absorption, as well as to a better resistance to soil pathogens under field conditions.

\section{CONCLUSIONS}

The main conclusions regarding the use of a new hybrid rootstock in promoting the grafted plant rate and watermelon yield are as follows:

1. The use of 'Wanzhen No. 2' significantly increased the survival rate of grafted seedlings in three watermelon varieties cultivated in three different locations. The insert- ing depth of the grafted seedlings affected root growth, and the most suitable depth was 2-3 cm for 'Wanzhen No. 2' when it comes to root regeneration.

2. The use of the 'Wanzhen No. 2' rootstock increased watermelon yield, when compared to the non-grafted plant control. Fruits harvested from the 'Wanzhen No. 2' grafted plants had a higher fruit weight than those harvested from the non-grafted plants, without affecting the content of the soluble solids.

3. No difference in fruit quality was observed, when compared to the non-grafted control plants. No plant wilt diseases were detected in the three types of watermelon in which the plants were grafted onto the 'Wanzhen No. 2' rootstock.

4. Our study proved that the 'Wanzhen No. 2' rootstock had a strong affinity to the watermelon seedlings, suggesting that it was a suitable rootstock for grafted seedlings in watermelons.

5. The findings from our study not only provide new insights into the pumpkin rootstock but also contribute to the improvement of the grafting method in rootstocks, when compared to the bottle gourd that is currently being used.

Conflict of interest: No potential conflict of interest was reported by the authors.

\section{REFERENCES}

Dorey E., Fournier P., Léchaudel M., and Tixier P., 2016. Modeling sugar content of pineapple under agro-climatic conditions on Reunion Island. Eur. J. Agron., 73, 64-72.

Edelstein M., Tyutyunik J., Fallik E., Meir A., Tadmor Y., and Cohen R., 2014. Horticultural evaluation of exotic watermelon germplasm as potential rootstocks. Sci. Hortic., 165, 196-202.

Hu B., Bennett M.A., and Kleinhenz M.D., 2016. A new method to estimate vegetable seedling vigor, piloted with tomato, for the use in grafting and other contexts. HortTechnol., 26, 767-775.

Huang Y., Jiao Y., Nawaz M.A., Chen C., Liu L., Lu Z., Kong Q., Cheng F., and Bie Z., 2016. Improving the magnesium uptake, photosynthesis and antioxidant enzyme activities of watermelon by grafting onto the pumpkin rootstock under low magnesium. Plant Soil, 409, 229-246.

Huang Y., Tang R., Cao Q., and Bie Z., 2009. Improving the fruit yield and quality of cucumber by grafting onto the salt tolerant rootstock under $\mathrm{NaCl}$ stress. Sci Hortic., 122, 26-31.

Huitrón-Ramírez M.V., Ricárdez-Salinas M., and CamachoFerre F., 2009. Influence of grafted watermelon plant density on yield and quality in soil infested with a melon necrotic spot virus. HortSci., 44, 1838-1841.

King S.R., Davis A.R., Liu W., and Levi A., 2008. Grafting for disease resistance. HortSci., 43, 1673-1676.

King S.R., Davis A.R., Zhang X., and Crosby K., 2010. Genetics, breeding and selection of rootstocks for Solanaceae and Cucurbitaceae. Sci. Hortic., 127, 106-111. 
Kolayli S., Kara M., Tezcan F., Erim F.B., Sahin H., Ulusoy E., and Aliyazicioglu R., 2010. A comparative study of chemical and biochemical properties of different melon cultivars: standard, hybrid and grafted melons. J. Agri. Food Chem., 58, 9764-9769.

Lee JM., Kubota C., Tsao S.J., Bie Z., Echevarria P.H., Morra L., and Oda M., 2010. Current status of vegetable grafting: Diffusion, grafting techniques, automation. Sci. Hortic., 127, 93-105.

Ling G., Shang Q., Len Y., and Zhang Z., 2012. Plug seedling substrate of vegetables. The standard of Agriculture Ministry in China: ICS 65.020 .020 B005, NY/T 2118-2012.

Louws F.J., Rivard C.L., and Kubota C., 2010. Grafting fruiting vegetables to manage soil-borne pathogens, foliar pathogens, arthropods and weeds. Sci. Hortic., 127, 127-146.

Lowe I., Cantu D., and Dubcovsky J., 2011. Durable resistance to wheat rusts: integrating systems biology and traditional phenotype-based research methods to guide the deployment of resistance genes. Euphytica, 179, 69-79.

Miguel A., Maroto J.V., San Bautista A., Baixauli C., Cebolla V., Pascual B., López S., and Guardiola J.L., 2004. The grafting of triploid watermelon is an advantageous alternative to soil fumigation by methyl bromide for the control of Fusarium wilt. Sci. Hortic., 103, 9-17.

Mo Y., Wang Y., Yang R., Zheng J., Liu C., Li H., Ma J., Zhang Y., Wei C., and Zhang X., 2016. Regulation of plant growth, photosynthesis, antioxidation and osmosis by an arbuscular mycorrhizal fungus in watermelon seedlings under well-watered and drought conditions. Front. Plant Sci., 7, 1-15.

Mohamed F.H., El-Hamed K.E.A., Elwan M.W.M., and Hussien M.N.E., 2014. Evaluation of different grafting methods and rootstocks in watermelons grown in Egypt. Sci. Hortic., 168, 145-150.

Proietti S., Rouphael Y., Colla G., Cardarelli M., De Agazio M., Zacchini M., Rea E., Moscatello S., and Battistelli A., 2008. Fruit quality of mini-watermelon as affected by grafting and irrigation regimes. J. Sci. Food Agri., 88, 1107-1114.

Reig G., Mestre L., Betrán J.A., Pinochet J., and Moreno M.Á., 2016. Agronomic and physicochemical fruit properties of the 'Big Top' nectarine budded on peach and plum based rootstocks in Mediterranean conditions. Sci. Hortic., 210, 85-92.

Ren Y., Di J., Gong G., Zhang H., Guo S., Zhang J., and Xu Y., 2015. Genetic analysis and chromosome mapping of resistance to Fusarium oxysporum f. sp. niveum (FON) race 1 and race 2 in watermelon (Citrullus lanatus L.). Mol. Breeding, 35, 1-9.
Ren Y., Zhao H., Kou Q., Jiang J., Guo S., Zhang H., Hou W., Zou X., Sun H., Gong G., Levi A., and Xu Y., 2012. A high resolution genetic map anchoring scaffolds of the sequenced watermelon genome. Plos One, 7: e29453.

Romano D. and Paratore A., 2001. Effects of grafting on tomato and eggplant. Acta. Hortic., 559, 149-153.

Savvas D., Colla G., Rouphael Y., and Schwarz D., 2010. Amelioration of heavy metal and nutrient stress in fruit vegetables by grafting. Sci. Hortic., 127, 156-161.

Trionfetti N.P., Colla G., Granati E., Temperini O., Crinò P., and Saccardo F., 2002. Rootstock resistance to fusarium wilt, and its effect on fruit yield and quality of two muskmelon cultivars. Sci. Hortic., 93, 281-288.

Turhan A., Ozmen N., Kuscu H., Serbeci M.S., and Seniz V., 2012. Influence of rootstocks on the yield, fruit characteristics and quality of watermelon. Horti. Environ. Biotechnol., 53, 336-341.

Vu N.T., Xu Z.H., Kim Y.S., Kang H.M., and Kim I.S., 2014. Effect of nursery environmental condition and different cultivars on the survival rate of grafted tomato seedlings. Acta. Hortic., 1037, 765-770.

Wang P.C., Fang L., Yan C.S., Jiang H.K., Tian H.M., Wan Y., Zhang J., and Zhang Q.A., 2015. Present situation and countermeasures of grafted seedling production in the Anhui province. Acta. Hortic., 1086, 41-50.

Xing W.W., Li L., Gao P., Li H., Shao Q.S., Shu S., Sun J., and Guo S.R., 2015. Effects of grafting with pumpkin rootstocks on carbohydrate metabolism in cucumber seedlings under $\mathrm{Ca}\left(\mathrm{NO}_{3}\right)^{2}$ stress. Plant Physiol. Biochem., 87, 124-132.

Yang X., Hu X., Zhang M., Xu J., Ren R., Liu G., Yao X., and Chen X., 2016. Effect of low night temperature on the graft union formation in watermelon grafted onto the bottle gourd rootstock. Sci. Hortic., 212, 29-34.

Yang Y., Lu X., Yan B., Li B., Sun J., Guo S., and Tezuka T., 2013. Bottle gourd rootstock-grafting affects nitrogen metabolism in $\mathrm{NaCl}$-stressed watermelon leaves and enhances short-term salt tolerance. J. Plant Physiol., 170, 653-661.

Yetışır H., Sari N., and Yücel S., 2003. Rootstock resistance to Fusarium wilt and its effect on watermelon fruit yield and quality. Phytoparasitica, 31, 163-169.

Zhu S., Vossen J.H., Bergervoet M., Nijenhuis M., Kodde L., Kessel G.J.T., Vleeshouwers V., Visser R.G.F., and Jacobsen E., 2015. An updated conventional and novel GM potato late blight $\mathrm{R}$ gene differential set for virulence monitoring of Phytophthora infestans. Euphytica, 202, 219-234 

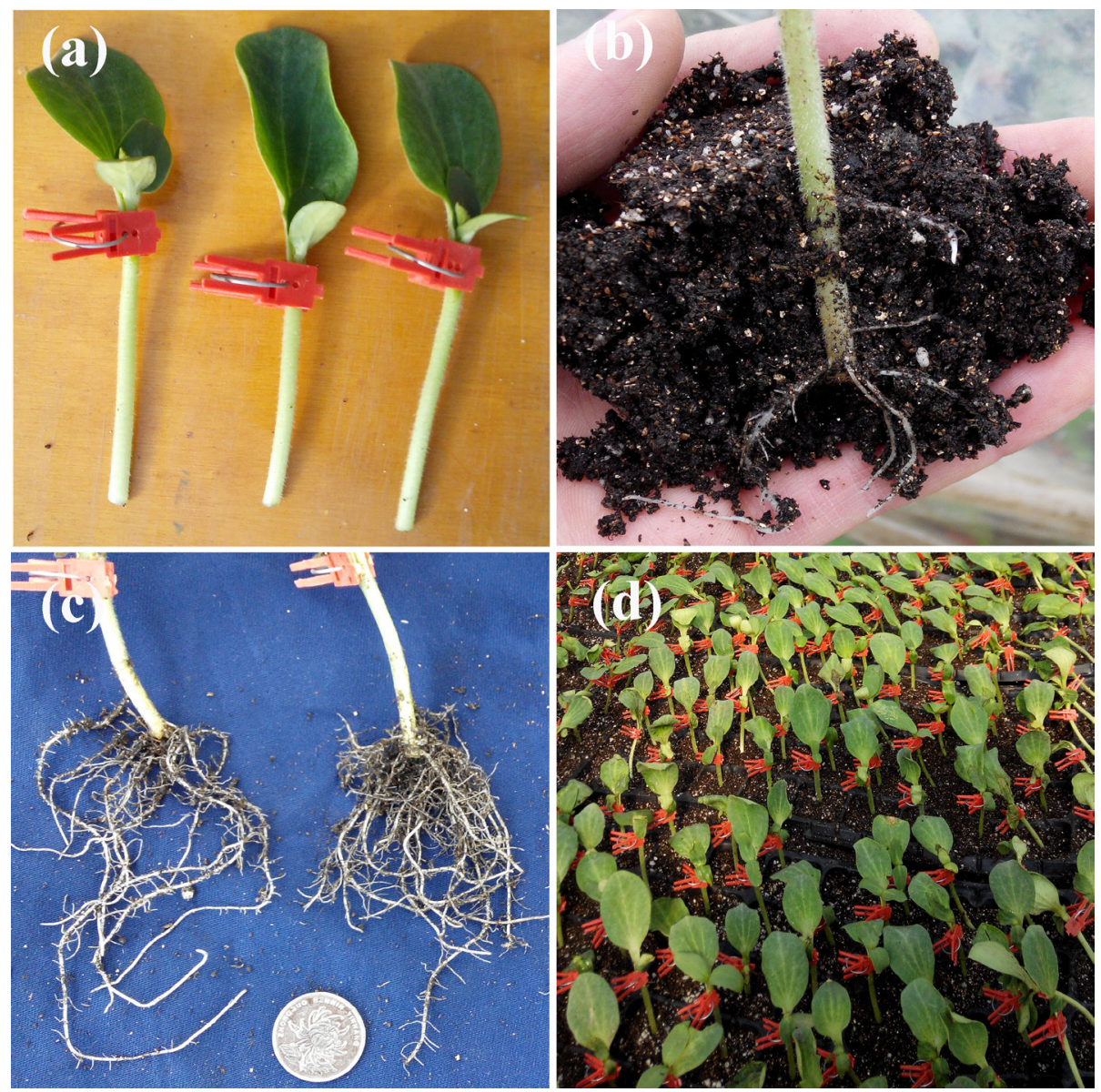

Fig. S1. Photos of the grafted plants and re-germinated roots in the experiment. (a) Grafted plants with double-root-cutting; the 'WZ2' root was cut and the scion was clipped together by using the tongue slide method; (b) a re-germinated root of the 'WZ2'rootstock after 7 days; (c) a developed root of 'WZ2' after 15 days; (d) a view of the grafted seedlings inserted in the substrate. 